\section{Pneumoperitoneum caused by gastroscopy in a jaundiced patient treated endoscopically after initial percutaneous approach}

A 78-year-old man with neoplastic obstructive jaundice, who had undergone placement of a percutaneous drainage catheter (10-Fr internal/external catheter), was admitted for endoscopic retrograde cholangiopancreatography (ERCP). The cholangiogram revealed an irregular stricture of the distal common bile duct. A hydrophilic guide wire was inserted across the transhepatic drainage and recovered endoscopically; we removed the percutaneous drain, performed a biliary sphincterotomy, and inserted an 8-cm Zilver metallic stent (Wilson-Cook Medical Inc., Winston-Salem, North Carolina, USA). Three days after ERCP, the patient underwent esophagogastroduodenoscopy with duodenal biopsies taken for histological analysis; the latter identified an adenocarcinoma. Immediately after esophagogastroduodenoscopy the patient developed acute abdominal pain. A CT scan ( $\odot$ Fig. 1 ) revealed free air in the abdomen and ascites around the spleen, in the right laterocolic space, and among the jejunal loops. The patient underwent urgent laparotomy but no bowel or biliary perforation was revealed. Intraoperative cholangiography showed correct working of the biliary stent, but free passage of contrast medium was observed through a fistula where the percutaneous drain had previously been. A cholecystectomy was performed and a $\mathrm{T}$ tube left in place. The postoperative course was normal. It would appear that air inflation during the esophagogastroduodenoscopy had facilitated the passage of air from the duodenum to the peritoneum across the fistula created by the percuta-

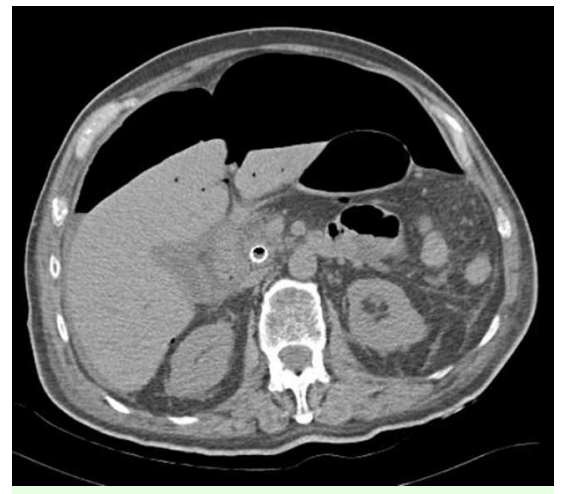

Fig. 1 In a patient with a biliocutaneous fistula after percutaneous drainage, CT scan shows free air in the abdomen and ascites around the spleen and liver, in the right laterocolic space, and among the jejunal loops. The biliary stent is also visible.

neous drain; the ascitic liquid, leaving a distance between the hepatic surface and the abdominal wall, made development of the pneumoperitoneum even easier, because of the incomplete consolidation of the biliocutaneous fistula.

In patients with a biliocutaneous fistula after percutaneous drainage, early endoscopic examination with insufflation of air can facilitate free passage of air into the abdomen from the fistula. Ascites may facilitate this process further; if ascites is present, therefore, it may be useful to leave in place an external biliary drain after placement of the stent to allow the fistula to consolidate. Definitive removal of the external drain may be more safely carried out 3-5 days after the endoscopic procedure.
Endoscopy_UCTN_Code_CPL_1AK_2AD

Endoscopy_UCTN_Code_CPL_1AK_2AH

M. Pandolfi ${ }^{1}$, R. Rea ${ }^{1}$, M. Martino ${ }^{1}$, P. Crucitti ${ }^{2}$, F. M. Di Matteo ${ }^{1}$,

G. Costamagna ${ }^{3}$, A. Gabbrielli ${ }^{1}$

Digestive Diseases Department, Campus Bio-Medico University, Rome, Italy

2 Department of General Surgery, Campus Bio-Medico University, Rome, Italy Surgical Endoscopy Unit, “A. Gemelli” Catholic University, Rome, Italy

Bibliography

DOI 10.1055/s-0028-1103445

Endoscopy 2009; 41: E16

(c) Georg Thieme Verlag KG Stuttgart · New York . ISSN 0013-726X

Corresponding author

M. Pandolfi, MD

Digestive Diseases Department Campus Bio-Medico University Via Alvaro del Portillo 200 Rome 00128

Italy

Fax: +39-06-225411658

m.pandolfi@unicampus.it 